\title{
Profiling of Somatic Mutations in Phaeochromocytoma and Paraganglioma by Targeted Next Generation Sequencing Analysis
}

\author{
Andrea Luchetti, ${ }^{1,2}$ Diana Walsh, ${ }^{3}$ Fay Rodger, ${ }^{2}$ Graeme Clark, ${ }^{1}$ Tom Martin, ${ }^{4}$ \\ Richard Irving, ${ }^{4}$ Mario Sanna, ${ }^{5,6}$ Masahiro Yao, ${ }^{7}$ Mercedes Robledo, ${ }^{8,9}$ \\ Hartmut P. H. Neumann, ${ }^{10}$ Emma R. Woodward, ${ }^{3,11}$ Farida Latif, ${ }^{3}$ Stephen Abbs, ${ }^{2}$ \\ Howard Martin, ${ }^{2}$ and Eamonn R. Maher ${ }^{1,2,3}$ \\ ${ }^{1}$ Department of Medical Genetics, University of Cambridge, Cambridge Biomedical Campus, Cambridge CB2 0QQ, UK \\ ${ }^{2}$ Cambridge NIHR Biomedical Research Centre, Addenbrooke's Hospital, Cambridge CB2 OQQ, UK \\ ${ }^{3}$ Centre for Rare Diseases and Personalised Medicine, University of Birmingham, Birmingham B15 2TT, UK \\ ${ }^{4}$ Queen Elizabeth Hospital, Queen Elizabeth Medical Centre, Birmingham B15 2TH, UK \\ ${ }^{5}$ Department of Otology \& Skull Base Surgery, Gruppo Otologico, Via Antonio Emmanueli 42, 29121 Piacenza, Italy \\ ${ }^{6}$ Department of Medical, Oral and Biotechnological Sciences, G. d’Annunzio University, Via dei Vestini 1, 66100 Chieti, Italy \\ ${ }^{7}$ Department of Urology, Yokohama City University School of Medicine, 3-9 Fukuura, Kanazawa Ward, Yokohama, \\ Kanagawa 236-0004, Japan \\ ${ }^{8}$ Hereditary Endocrine Cancer Group, Spanish National Cancer Research Centre (CNIO), Madrid, Spain \\ ${ }^{9}$ ISCIII Center for Biomedical Research on Rare Diseases (CIBERER), Madrid, Spain \\ ${ }^{10}$ Section of Preventive Medicine, Department of Nephrology, Albert Ludwigs University of Freiburg, Hugstetter Strasse 55, \\ 79106 Freiburg, Germany \\ ${ }^{11}$ Department of Clinical Genetics, Birmingham Women's Hospital, Birmingham B15 2TG, UK
}

Correspondence should be addressed to Eamonn R. Maher; erm1000@medschl.cam.ac.uk

Received 27 June 2014; Revised 23 August 2014; Accepted 26 August 2014

Academic Editor: Claudio Letizia

Copyright (C) 2015 Andrea Luchetti et al. This is an open access article distributed under the Creative Commons Attribution License, which permits unrestricted use, distribution, and reproduction in any medium, provided the original work is properly cited.

At least 12 genes (FH, HIF2A, MAX, NF1, RET, SDHA, SDHB, SDHC, SDHD, SDHAF2, TMEM127, and VHL) have been implicated in inherited predisposition to phaeochromocytoma (PCC), paraganglioma (PGL), or head and neck paraganglioma (HNPGL) and a germline mutation may be detected in more than $30 \%$ of cases. Knowledge of somatic mutations contributing to PCC/PGL/HNPGL pathogenesis has received less attention though mutations in HRAS, HIF2A, NF1, RET, and VHL have been reported. To further elucidate the role of somatic mutation in PCC/PGL/HNPGL tumourigenesis, we employed a next generation sequencing strategy to analyse "mutation hotspots" in 50 human cancer genes. Mutations were identified for HRAS (c.37G>C; p.G13R and c.182A>G; p.Q61R) in 7.1\% (6/85); for BRAF (c.1799T>A; p.V600E) in 1.2\% (1/85) of tumours; and for TP53 (c.1010G>A; p.R337H) in 2.35\% $(2 / 85)$ of cases. Twenty-one tumours harboured mutations in inherited PCC/PGL/HNPGL genes and no HRAS, BRAF, or TP53 mutations occurred in this group. Combining our data with previous reports of HRAS mutations in PCC/PGL we find that the mean frequency of HRAS/BRAF mutations in sporadic PCC/PGL is 8.9\% (24/269) and in PCC/PGL with an inherited gene mutation $0 \%$ (0/148) suggesting that $H R A S / B R A F$ mutations and inherited PCC/PGL genes mutations might be mutually exclusive. We report the first evidence for BRAF mutations in the pathogenesis of PCC/PGL/HNPGL.

\section{Introduction}

Phaeochromocytoma (PCC) and paraganglioma (PGL) are neuroendocrine tumours deriving from chromaffin cells of the medulla of the adrenal glands or from extra-adrenal chromaffin tissue like ganglia of the sympathetic nervous system, respectively. Approximately $10 \%$ of tumours are malignant but the most common presentation results from 
the cardiovascular effects of catecholamine hypersecretion that causes hypertension, tachycardia, excessive sweating, and/or anxiety. The majority of PCC/PGL occur sporadically but although only about $10 \%$ of cases have a family history, more than a third of cases harbour a germline mutation in one of the 12 inherited PCC/PGL genes (FH, HIF2A, MAX, NF1, RET, SDHA, SDHB, SDHC, SDHD, SDHAF2, TMEM127, and $V H L$ ) that have been shown to be mutated in multiple families and some of these genes are also somatically mutated in sporadic PCC/PGL [1-8]. Germline mutations in most of these genes may also cause head and neck paraganglioma (HNPGL) which are derived from parasympathetic nervous system ganglia and are nonsecretory [9-12]. It appears that disruption of multiple cellular signalling pathways is implicated in PCC/PGL/HNPGL tumourigenesis. Thus known inherited predisposition gene products belong to multiple functional classes including kinase receptor and signalling regulators (RET and NF1), transcription factors (MAX), energy metabolism components $(F H, S D H$-subunits $A, B$, $C$, and $D$, and cofactor SDHAF2), constituents of the cellular response to hypoxia (VHL and HIF2A/EPAS1), and endosomal signalling (TMEM127) [1-8, 13-15]. Nevertheless, gene expression studies have suggested that most PCCs and PGLs can be classified into two distinct groups (cluster 1 and cluster 2) by transcription profiling: cluster 1 includes tumours that harbour mutations in genes linked to the hypoxic gene response (VHL, HIF2A, SDHA, SDHB, SDHC, and $S D H D$ ) and cluster 2 contains tumours harbouring mutations in genes that are involved in the kinase signalling characterized by the activation of the PIK3/AKT/mTOR and RAS/RAF/ERK pathways (RET, NF1, TMEM127, MAX, and HRAS) [14-18].

The last 10 years have seen considerable progress in identifying the genetic basis of inherited PCC/PGL/HNPGL, but relatively less progress has been made with respect to understanding the somatic mutations that underlie tumour initiation and progression in these tumour types. Somatic mutations in inherited PCC/PGL genes can be detected in $\sim 25-30 \%$ of the sporadic tumours (mainly involving RET, $V H L, N F 1, M A X$, and HIF2A genes). Recently, an exome resequencing study led to the identification of somatic HRAS activating mutations in sporadic PCC/PGLs [19]. Subsequently, this finding was confirmed and the overall frequency of somatic HRAS mutations has been estimated at about 7\% [20]. However, to date HRAS analysis in PCC/PGL has mostly been performed by Sanger sequencing and the frequency of HRAS mutations might have been underestimated because of the lower sensitivity of Sanger sequencing analysis, compared to next generation sequencing approaches, to detect mosaic mutations [21]. Furthermore, in contrast to many other tumour types, there is little information available on many of the genes most commonly mutated in human neoplasia. We therefore investigated, in tumour samples, the frequency of mutations in critical regions of 50 human cancer genes by next generation sequencing in a large series $(n=85)$ of inherited and sporadic PCC/PGL/HNPGL.

\section{Materials and Methods}

2.1. Subjects. Tumour material from 85 patients with PCC $(n=60)$, PGL $(n=5)$, or HNPGL $(n=20)$ was collected for analysis. 21 patients were known to harbour a germline inherited gene mutation $(V H L=10, R E T=3, N F 1=1, S D H B$ $=5, S D H C=1$, and $S D H D=1)$ and 64 cases were sporadic. All patients gave informed consent and the study was approved by the South Birmingham Ethics Committee.

\subsection{Next Generation Sequencing (NGS)}

2.2.1. Technical Assessment. To investigate the sensitivity of somatic mutation detection, a dilution series of two well characterized DNA samples was created. A sample bearing HRAS (c.81T>C) and one a PIK3CA (c.1173A>G) were titrated together to create serial dilutions containing allelic frequencies ranging from $50 \%$ to $0.1 \%$. Each dilution was amplified using the Ion AmpliSeq Cancer Hotspot Panel v2 (Life Technologies, UK). Amplicons underwent library preparation according to protocol. Before emulsion PCR each point of the titration curve was identified by using a different Ion Xpress Barcode. Subsequently library was run on 318 chip v2 (Life Technologies, UK) on the Ion PGM (Life Technologies, UK). The output reads from the chip were processed using the Torrent browser suite software (v.4.0.2).

2.2.2. Sample Sequencing. DNA was isolated from both tumour material and peripheral blood using standard methodology. Genomic DNA (gDNA) samples were qualitychecked on DNA NanoDrop 1000 considering acceptable absorbance ratio greater than 1.7 for both 260/280 and $230 / 260 \mathrm{~nm}$. Each sample was then quantified with the Qubit2.0 fluorometer (Life Technologies, UK) by using the Quant-IT dsDNA BR Assay (Life Technologies, UK). For the AmpliSeq Library, 10 ng of gDNA was used for library generation. Libraries were indexed using the Ion Xpress Barcode Adapter Kit and quantified using the Quant-IT dsDNA HS Assay (Life Technologies, UK) on Qubit 2.0. Appropriate dilutions were performed based on amplicon concentration at the $80-125$ bp range. Twenty $\mathrm{pM}$ of individual indexed amplicon libraries were pooled for emulsion PCR and 16 samples were sequenced on the Ion Torrent PGM platform using the $318 \mathrm{v} 2$ chip (Life Technologies, UK). Mean coverage for each sample was over 1000x. Sequence reads were mapped against the human reference genome (hg19) with the Torrent Mapping Alignment Program (TMAP) using the default software settings. Output was restricted to the targeted regions as defined by the sequence capture design BED file, and SNPs and INDELs were characterized as being significantly different from the reference sequence if the variant to reference base frequency was greater than $5 \%$. All identified variants within a particular sample were saved as variant call format file (VCF version 4.1). VCFs were examined with the online tool "Ingenuity Variant Analysis" (Qiagen) for variant annotation and prediction of variant effects on genes. In addition, BAM files were inspected manually in order to remove likely artefactual variants (i.e., close to homopolymers) and to detect any mutations in 
TABLE 1: Mutations detected in inherited PCC/PGL/HNPGL genes.

\begin{tabular}{lccc}
\hline Tumour ID & Type of tumour & Gene & Mutation type \\
\hline P1 & PCC & $R E T$ & c.1900T $>$ C \\
P2 & PCC & $R E T$ & c.1901G $>$ A \\
P3 & PCC & $R E T$ & c.2753T $>$ C \\
P4 & PCC & $V H L$ & c.241C $>$ T \\
P5 & PCC & $V H L$ & c.292T $>$ C \\
P6 & PCC & $V H L$ & c.292T $>$ C \\
P7 & PCC & $V H L$ & c.292T $>$ C \\
P8 & PCC & $V H L$ & c.292T $>$ C \\
P9 & PCC & $V H L$ & c.292T $>$ C \\
P10 & PCC & $V H L$ & c.292T $>$ C \\
P11 & PCC & $V H L$ & c.292T $>$ C \\
P12 & PCC & $V H L$ & c.292T $>$ C \\
P13 & PCC & $V H L$ & c.374A $>C$ \\
P14 & PCC & $S D H B$ & c.470delT \\
\hline
\end{tabular}

known driver genes, especially insertions and deletions that were not called by the Ion Torrent software.

2.3. Sanger Sequencing. To confirm NGS results, fragments of hotspot codons in HRAS (exons 2 and 3), BRAF (exon 15), and TP53 (exon 10) were amplified, in both tumour and constitutional (blood) DNA, by PCR and sequenced with automated Sanger sequencing. Primers sequences and PCR conditions are available on request.

2.4. Statistical Analysis. Patients with mutated HRAS and $B R A F$ were compared to patients with negative genetic screening.

$P$ values $<0.05$ were considered statistically significant.

\section{Results}

3.1. Technical Assessment of Next Generation Sequencing Assay. The analytical sensitivity of the AmpliSeq Hotspot panel was determined using serial dilutions of tumour DNA carrying PIK3CA and HRAS mutations. This demonstrated that it was possible to reliably detect mutations at $1 \%$ allele frequency (data not shown). Sequence coverage was assessed considering the number and distribution of reads that were present in the target DNA regions. Each sample had approximately 317000 mapped reads with a mean read length of $107 \mathrm{bp}$ that generates approximately $23 \mathrm{Mb}$ of sequence with depth of coverage of 1400 reads.

\subsection{Detection of Mutations in Inherited PCC/PGL/HNPGL} Genes. 21 patients were known to harbour a germline mutation in an inherited PCC/PGL gene $(V H L=10, R E T=3, N F 1=$ $1, S D H B=5, S D H C=1$, and $S D H D=1) .13$ of these mutations $(\operatorname{RET}(n=3)$ and VHL $(n=10))$ were detected by NGS in 13 tumours (see Table 1 ) and 8 patients had a clinical or previous molecular diagnosis of a germline inherited PCC/PGL gene mutation (NF1 $(n=1), \operatorname{SDHB}(n=5)$, SDHC $(n=1)$, and $\operatorname{SDHD}(n=1))$ that was not covered by the NGS assay. No mutations in inherited PCC/PGL genes were detected in the 64 sporadic tumours.

3.3. Detection of Activating Mutations in Protooncogenes and Tumour Suppressor Genes. Six tumours (PCC $=6 / 60$, PGL $=0 / 5$, and $\mathrm{HNPGL}=0 / 20$ ) harboured an activating missense mutation in the HRAS hotspot region of codons 13 and 61 (c.37G>C; p.G13R = 1 and c.182A>G; p.Q61R $=5)$, giving an overall frequency of $7.1 \%(6 / 85,95 \% \mathrm{CI}$ $=2.63 \%-14.73 \%)$. In each case the somatic status of the mutations was confirmed when the detected mutation was absent in matched constitutional DNA (blood) (Table 2). In one PCC tumour (1/85) an activating BRAF mutation was identified (c.1799T >A; p.V600E, 1.2\%, 95\% CI = 0\%-6.38\%) (Table 2). In two tumours a missense mutation (c.1010G $>A$; p.R337H, 2/85, 2.4\%, 95\% CI $=0.29 \%-8.2 \%$ ) occurring in the tetramerisation domain of TP53 protein was identified (Table 2).

3.4. Exclusion of Mutations in Protooncogene Hotspots. No mutations were detected at hotspot mutation regions in 11 oncogenes frequently mutated in human cancer (AKT, MET, PIK3CA, KRAS, NRAS, IDH1, IDH2, NOTCH, SMO, ABL, and $E G F R)$.

3.5. Relationship between Clinical Status and HRAS Mutations. We investigated the relationships between HRAS mutation and tumour location and presence or absence of an inherited PCC/PGL/HNPGL gene mutation. The frequency of HRAS mutations in PCC, PGL, and HNPGL was 6/60 (10\%, $95 \% \mathrm{CI}=3.76 \%-20.51 \%), 0 / 5(0 \%, 95 \% \mathrm{CI}=0 \%-52.18 \%)$, and $0 / 20(0 \%, 95 \% \mathrm{CI}=0 \%-16.84 \%)$, respectively. Among 21 tumours from patients with known inherited disease and/or detectable mutation in inherited PCC/PGL/HNPGL genes there were no HRAS mutations (0\%, 95\% CI =0\%-16.11\%) whereas a mutation was present in $6 / 64(9.4 \%, 95 \% \mathrm{CI}=$ $3.52 \%-19.30 \%)$ of patients without a clinical diagnosis of inherited PCC/PGL/HNPGL or a detectable mutation in an inherited PCC/PGL/HNPGL gene $(P=0.33)$.

To further investigate possible relationships between these attributes we combined our data for HRAS mutation status with 18 mutations in 353 PCC/PGL/HNPGL from two previously published studies (Tables 3 and 4) $[19,20]$. Meta-analysis results showed that the overall prevalence of HRAS mutations in the cohort of PCC/PGL is 5.48\% (24/438, $95 \% \mathrm{CI}=3.54 \%-8.04 \%)$ increasing to $8.9 \%(24 / 269,95 \%$ $\mathrm{CI}=5.80 \%-12.98 \%)$ considering cases without an inherited PCC/PGL gene mutation and to $9.87 \%$ (23/233, 95\% CI = $6.36 \%-14.44 \%)$ considering only PCC samples without an inherited PCC/PGL gene. In PCC/PGL with an inherited gene mutation the $H R A S$ mutation frequency was $0 \%(0 / 148$, 95\% CI $=0 \%-2.46 \%)(\mathrm{PCC} / \mathrm{PGL}$ unknown mutation versus PCC/PGL known mutation, $P=0.0001)$.

\section{Discussion}

A wide repertoire of genetic and epigenetic events can be implicated in human neoplasia. Previous studies have 
TABLE 2: Oncogene mutations identified in next generation sequencing analysis of 85 PCC/PGL/HNPGL.

\begin{tabular}{|c|c|c|c|c|c|c|}
\hline Tumour ID & Clinical diagnosis & Type of tumour & Gene & Codon change & Aminoacid change & Allele frequency \\
\hline P19 & Phaeochromocytoma & PCC & HRAS & c. $37 \mathrm{G}>\mathrm{C}$ & $\mathrm{G} 13 \mathrm{R}$ & $72 \%$ \\
\hline P20 & Phaeochromocytoma & PCC & HRAS & c. $182 \mathrm{~A}>\mathrm{G}$ & Q61R & $36 \%$ \\
\hline P21 & Phaeochromocytoma & PCC & HRAS & c. $182 \mathrm{~A}>\mathrm{G}$ & Q61R & $27 \%$ \\
\hline P22 & Phaeochromocytoma & PCC & HRAS & c. $182 \mathrm{~A}>\mathrm{G}$ & Q61R & $40 \%$ \\
\hline P23 & Phaeochromocytoma & PCC & HRAS & c. $182 \mathrm{~A}>\mathrm{G}$ & Q61R & $50 \%$ \\
\hline P24 & Phaeochromocytoma & PCC & HRAS & c. $182 \mathrm{~A}>\mathrm{G}$ & Q61R & $26 \%$ \\
\hline P25 & Phaeochromocytoma & PCC & $B R A F$ & c.1799T $>A$ & $\mathrm{~V} 600 \mathrm{E}$ & $10 \%$ \\
\hline P26 & Phaeochromocytoma & PCC & TP53 & c.1010G $>A$ & R337H & $4 \%$ \\
\hline P27 & Phaeochromocytoma & PCC & TP53 & c.1010G $>A$ & R337H & $21 \%$ \\
\hline
\end{tabular}

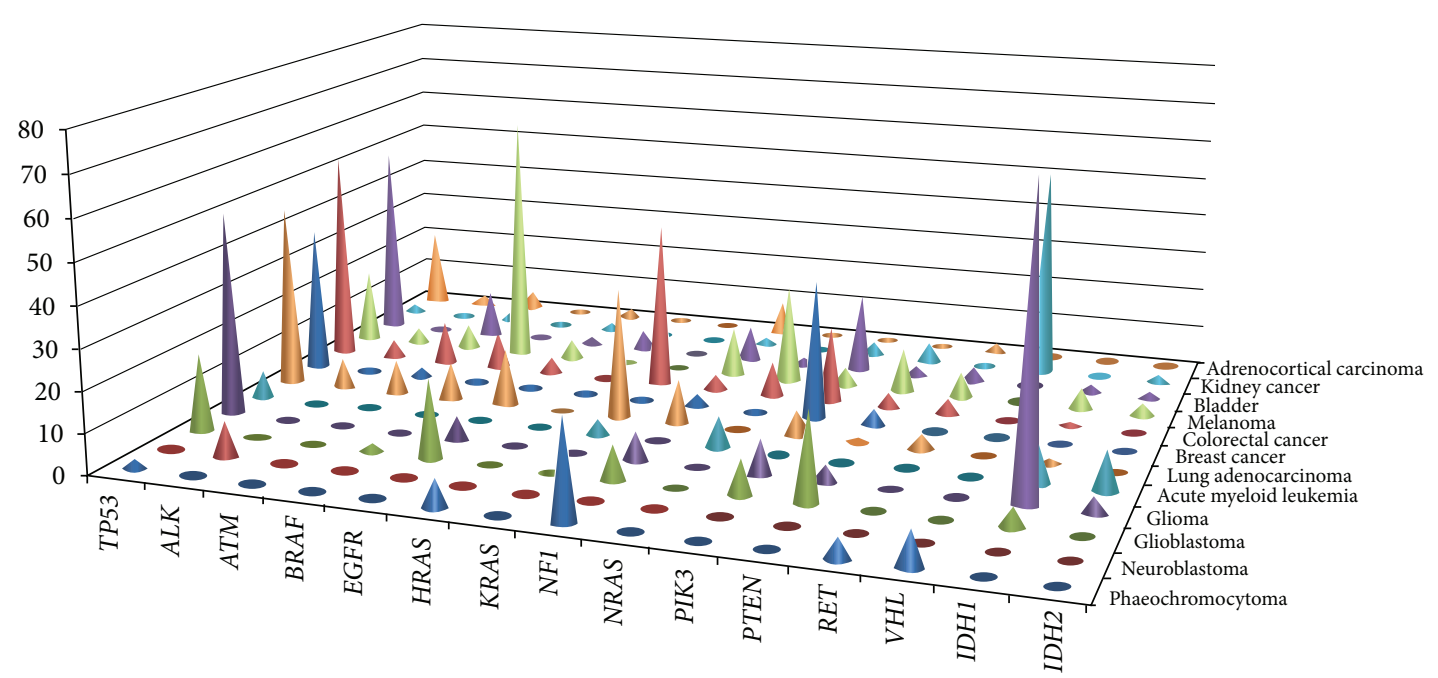

FIGURE 1: Comparison of somatic variant frequencies in multiple cancer genes in different cancer types.

demonstrated that tumour suppressor gene (TSG) inactivation and oncogene activation in PCC/PGL may result from somatic copy number abnormalities (SCNA), intragenic mutations, and epigenetic silencing of transcription by promoter methylation [22]. Common copy number changes in PCC include loss of chromosomes 1p, 3q, 3p, 11p, 11q, 6q, 17p, and 22 [23-27] and gain of chromosomes 9q, 17q, 19p13.3, and $20 q[28,29]$. Epigenetic inactivation of candidate TSGs has been reported relatively frequently in PCC/PGL. Thus promoter methylation of candidate TSGs including RASSF1A, FLIP, TSP1, DCR1, DCR2, DR4, DR5, CASP8, and HIC1 was reported at a frequency of $>20 \%$ of tumours analysed [30-32]. Until recently, investigations of patterns of somatic mutations in PCC/PGL/HNPGL had concentrated on analysing genes known to be associated with inherited PCC/PGL/HNPGL. Thus NF1, VHL, RET, and MAX germline and somatic mutations have been reported in 3-25\%, 13-9\%, 5-5\%, and 1-3\% of tumours, respectively [33]. Recently activating mutations in $H I F 2 A$ and HRAS were reported in a subset of these tumours $[6,7,19,20]$. Whilst HIF2A mutations may be found in multiple tumours from patients without a detectable germline mutation (suggesting low level constitutional mosaicism) or occasionally as a germline mutation [5], to date HRAS mutations have only been detected as somatic changes (germline HRAS mutations are associated with Costello syndrome but PCC/PGL/HNPGL are apparently not a feature of this disorder) [34]. The frequency of HRAS mutations in our cohort was similar to that in other recent studies. HRAS mutation hotspots at codons 13 and 61 affect the RAS GTP hydrolysis domain, leading to a constitutive activated state with resistance to upstream inhibitory proteins, such as neurofibromin (NF1 gene product). This overactive RAS signalling leads to increased activity of downstream effectors, most notably the RAS/RAF/ERK and PI3K/AKT/mTOR signalling pathways linked to increased cell proliferation and tumour formation [14-18]. The identification of HRAS mutations as a new pathogenetic driver in sporadic PCC opens up the possibility of new therapeutic approachesthough in most cases surgical removal seems likely to be the treatment of choice. BRAF mutations are found in multiple cancer types (Figure 1), notably those that are also associated with mutations in isoforms of RAS (i.e., malignant melanoma, colorectal cancer). Our results demonstrate for the first time a somatic BRAF mutation in PCC/PGL/HNPGL samples. The mutation detected is the most common $B R A F$ mutation found in human neoplasia and results from a $\mathrm{T}$ to A transversion at nucleotide 1799. c.1799T >A (p.V600E) mutant BRAF proteins are characterized by an increased kinase activity and have been demonstrated to induce cellular transformation in in vitro studies [35]. 


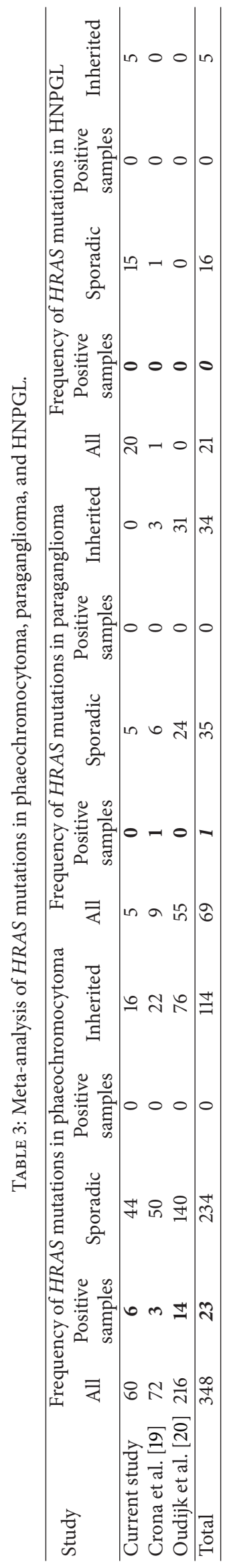


TABLE 4: HRAS mutations frequencies in phaeochromocytoma and paraganglioma.

\begin{tabular}{|c|c|c|c|c|c|c|c|c|c|}
\hline \multirow[t]{2}{*}{ Study } & \multicolumn{3}{|c|}{ Overall frequency of $H R A S$ mutations } & \multicolumn{3}{|c|}{$\begin{array}{c}\text { Frequency of } H R A S \text { in } \\
\text { PCC/PGL with unknown mutations }\end{array}$} & \multicolumn{3}{|c|}{$\begin{array}{l}\text { Frequency of } H R A S \text { in PCC } \\
\text { with unknown mutations }\end{array}$} \\
\hline & & All & Positive samples & & All & Positive samples & & All & Positive samples \\
\hline Current study & $7,06 \%$ & 85 & 6 & $12,24 \%$ & 49 & 6 & $13,95 \%$ & 43 & 6 \\
\hline Crona et al. [19] & $4,88 \%$ & 82 & 4 & $7,14 \%$ & 56 & 4 & $6,00 \%$ & 50 & 3 \\
\hline Oudijk et al. [20] & $5,17 \%$ & 271 & 14 & $8,54 \%$ & 164 & 14 & $10,00 \%$ & 140 & 14 \\
\hline Total & $5,48 \%$ & 438 & 24 & $8,92 \%$ & 269 & 24 & $9,87 \%$ & 233 & 23 \\
\hline
\end{tabular}

Since BRAF is involved in ERK kinase activation in the RAS/RAF/ERK signalling pathway, it will be interesting to further compare the biological behaviour of $H R A S$ and $B R A F$ mutated tumours in order to determine whether they have similar clinical characteristics and to determine if they will fall into the "cluster 2" group of gene expression patterns. PCC/PGLs with BRAF V600E mutations may be predicted to respond to BRAF and MEK inhibitors, such as vemurafenib and trametinib/selumetinib, better than wild type $B R A F$ tumours [36-39].

The presence of $B R A F$ and HRAS mutations in PCC/PGL suggests that activation of the RAS/RAF/ERK signalling pathway can be triggered by mutations at various levels in the pathway. An implementation of the panel used, including more genes of this pathway, could be useful to identify low frequency somatic variants in PCC/PGL. Loss of heterozygosity at $17 \mathrm{p} 13$ is frequent in many tumour types including breast [40], colon [41], and hepatocellular cancers [42] and phaeochromocytoma [22], with an occurrence ranging from $30 \%$ to $60 \%$. The tumour suppressor gene TP53 maps in this region and has been demonstrated to be implicated in the tumourigenesis process in different types of cancers. Loss of TP53 function could arise from epigenetic alterations allelic losses and mutational events. Due to its involvement in carcinogenesis, TP53 has been intensively studied in PCC/PGL but, despite frequent allele loss, TP53 mutations have been reported rarely [23, 43-45]. However, in our series, NGS results followed by direct DNA sequencing demonstrated the presence in two sporadic PCC samples of a somatic TP53 gene mutation (c.1010G>A; p.R337H) (Table 2). The R337H substitution has previously been extensively characterised as a founder germline mutation and the altered protein demonstrated to act as a conditional mutant that loses its function only when a small increase in intracellular $\mathrm{pH}$ occurs in cells. Initially, the c.1010G $>$ A (R337H) mutation was thought to predispose only to adrenal cortical carcinoma (ACC) (for which the penetrance of the allele has been estimated to be $\sim 10 \%$ and the increased risk was estimated at a 20,000-fold increase $[46,47]$ ); however, several studies have highlighted it in association with Li Fraumeni-like syndrome [48], breast cancer $[49,50]$, choroid plexus carcinoma, and osteosarcoma [51, 52]. To our knowledge, this is the first report on this mutation in sporadic phaeochromocytoma. By using an approach utilising targeted deep sequencing we were able to confidently detect the presence or absence of a large repertoire of hotspot mutations, paying particular attention to a set of them. The absence of mutations in ALK and NRAS was of particular interest (Figure 1) because mutations in these genes have been described in neuroblastoma $(\sim 9.2 \%$, $0.83 \%$ of cases, resp.; Figure 1 ) and some epigenetically inactivated candidate TSGs (i.e., RASSF1A, FLIP, CASP8, and HIC1) are common to both PCC/PGL and neuroblastoma. IDH1 and IDH2 mutations have been described in gliomas, leukaemia, and other malignancies (Figure 1) and may cause methylation abnormalities by a similar mechanism to that associated with SDH gene subunit mutations [53]. Therefore despite IDH1/IDH2 hotspot regions potentially representing candidate genes for somatic inactivation in PCC/PGL/HNPGL, no mutations were identified (Figure 1) [54]. Our findings are consistent with the hypothesis that mutations in HRAS and inherited PCC/PGL genes are mutually exclusive driver mutations (though comprehensive analysis of all inherited phaeochromocytoma/PGL genes has not been undertaken in all patients). If this hypothesis is correct, it can be suggested that HRAS profiling of PCC/PGL/HNPGL tumour material could aid the management of patients by enabling enhanced stratification of their risk of inherited disease. Furthermore, we suggest that molecular profiling should be expanded to include BRAF analysis. Currently histopathology cannot reliably predict the likelihood of malignancy in PCC/PGL/HNPGL whereas the presence of a germline SDHB mutation is associated with an increased risk of malignancy [55-57]. The identification, careful characterisation, and follow-up of cohorts of patients with $H R A S / B R A F$ mutation positive tumours could enable the natural history of such tumours (e.g., absence of malignant or recurrent disease) and so facilitate personalised management of patients with these tumours.

\section{Conflict of Interests}

The authors declare that there is no conflict of interests regarding the publication of this paper.

\section{Acknowledgments}

The authors thank the NIHR Cambridge BioResource and British Heart Foundation for financial support.

\section{References}

[1] M. Jafri and E. R. Maher, "The genetics of phaeochromocytoma: using clinical features to guide genetic testing," European Journal of Endocrinology, vol. 166, no. 2, pp. 151-158, 2012.

[2] A.-P. Gimenez-Roqueplo, P. L. Dahia, and M. Robledo, "An update on the genetics of paraganglioma, pheochromocytoma, 
and associated hereditary syndromes," Hormone and Metabolic Research, vol. 44, no. 5, pp. 328-333, 2012.

[3] H. P. Neumann, B. Bausch, S. R. McWhinney et al., "Germline mutations in nonsyndromic pheochromocytoma," The New England Journal of Medicine, vol. 346, no. 19, pp. 1459-1466, 2002.

[4] N. Burnichon, L. Vescovo, L. Amar et al., "Integrative genomic analysis reveals somatic mutations in pheochromocytoma and paraganglioma," Human Molecular Genetics, vol. 20, no. 20, pp. 3974-3985, 2011.

[5] N. Burnichon, A. Buffet, B. Parfait et al., "Somatic NF1 inactivation is a frequent event in sporadic pheochromocytoma," Human Molecular Genetics, vol. 21, no. 26, pp. 5397-5405, 2012.

[6] Z. Zhuang, C. Yang, F. Lorenzo et al., "Somatic HIF2A gain-offunction mutations in paraganglioma with polycythemia," The New England Journal of Medicine, vol. 367, no. 10, pp. 922-930, 2012.

[7] I. Comino-Méndez, A. A. de Cubas, C. Bernal et al., "Tumoral EPAS1 (HIF2A) mutations explain sporadic pheochromocytoma and paraganglioma in the absence of erythrocytosis," Human Molecular Genetics, vol. 22, no. 11, pp. 2169-2176, 2013.

[8] E. Rattenberry, L. Vialard, A. Yeung et al., "A comprehensive next generation sequencing-based genetic testing strategy to improve diagnosis of inherited pheochromocytoma and paraganglioma," Journal of Clinical Endocrinology and Metabolism, vol. 98, no. 7, pp. E1248-E1256, 2013.

[9] B. E. Baysal, R. E. Ferrell, J. E. Willett-Brozick et al., "Mutations in SDHD, a mitochondrial complex II gene, in hereditary paraganglioma," Science, vol. 287, no. 5454, pp. 848-851, 2000.

[10] S. Niemann and U. Müller, "Mutations in SDHC cause autosomal dominant paraganglioma, type 3," Nature Genetics, vol. 26, no. 3, pp. 268-270, 2000.

[11] D. Astuti, F. Latif, A. Dallol et al., "Gene mutations in the succinate dehydrogenase subunit SDHB cause susceptibility to familial pheochromocytoma and to familial paraganglioma," The American Journal of Human Genetics, vol. 69, no. 1, pp. 4954, 2001.

[12] N. Burnichon, J.-J. Brière, R. Libé et al., "SDHA is a tumor suppressor gene causing paraganglioma," Human Molecular Genetics, vol. 19, no. 15, pp. 3011-3020, 2010.

[13] G. Eisenhofer, T.-T. Huynh, K. Pacak et al., "Distinct gene expression profiles in norepinephrine- and epinephrineproducing hereditary and sporadic pheochromocytomas: activation of hypoxia-driven angiogenic pathways in von Hippel-Lindau syndrome," Endocrine-Related Cancer, vol. 11, no. 4, pp. 897-911, 2004.

[14] E. López-Jiménez, G. Gómez-López, L. J. Leandro-García et al., "Research resource: transcriptional profiling reveals different pseudohypoxic signatures in SDHB and VHL-related pheochromocytomas," Molecular Endocrinology, vol. 24, no. 12, pp. 2382-2391, 2010.

[15] P. L. Dahia, K. N. Ross, M. E. Wright et al., "AHIF1 $\alpha$ regulatory loop links hypoxia and mitochondrial signals in pheochromocytomas," PLoS Genetics, vol. 1, pp. 172-180, 2005.

[16] A. Vicha, Z. Musil, and K. Pacak, "Genetics of pheochromocytoma and paraganglioma syndromes: new advances and future treatment options," Current Opinion in Endocrinology, Diabetes and Obesity, vol. 20, no. 3, pp. 186-191, 2013.

[17] P. L. M. Dahia, K. Hao, J. Rogus et al., "Novel pheochromocytoma susceptibility loci identified by integrative genomics," Cancer Research, vol. 65, no. 21, pp. 9651-9658, 2005.
[18] J. Favier and A.-P. Gimenez-Roqueplo, "Pheochromocytomas: the (pseudo)-hypoxia hypothesis," Best Practice and Research: Clinical Endocrinology and Metabolism, vol. 24, no. 6, pp. 957968, 2010.

[19] J. Crona, A. Delgado Verdugo, R. Maharjan et al., "Somatic mutations in H-RAS in sporadic pheochromocytoma and paraganglioma identified by exome sequencing," Journal of Clinical Endocrinology and Metabolism, vol. 98, no. 7, pp. E1266-E1271, 2013.

[20] L. Oudijk, R. Ronald, R. R. de Krijger et al., "H-RAS mutations are restricted to sporadic pheochromocytomas lacking specific clinical or pathological features: data from a multi-institutional series," The Journal of Clinical Endocrinology and Metabolism, vol. 99, no. 7, pp. E1376-E1380, 2014.

[21] J. S. Ross and M. Cronin, "Whole cancer genome sequencing by next-generation methods," American Journal of Clinical Pathology, vol. 136, no. 4, pp. 527-539, 2011.

[22] A. Blanes, J. J. Sanchez-Carrillo, and S. J. Diaz-Cano, “Topographic molecular profile of pheochromocytomas: role of somatic down-regulation of mismatch repair," Journal of Clinical Endocrinology and Metabolism, vol. 91, no. 3, 2006.

[23] A. Cascon, S. Ruiz-Llorente, M. F. Fraga et al., "Genetic and epigenetic profile of sporadic pheochromocytomas," Journal of Medical Genetics, vol. 41, no. 3, article e30, 2004.

[24] J. Sandgren, R. Andersson, A. Rada-Iglesias et al., "Integrative epigenomic and genomic analysis of malignant pheochromocytoma," Experimental and Molecular Medicine, vol. 42, no. 7, pp. 484-502, 2010.

[25] A. Hering, M. Guratowska, P. Bucsky et al., "Characteristic genomic imbalances in pediatric pheochromocytoma," Genes, Chromosomes and Cancer, vol. 45, no. 6, pp. 602-607, 2006.

[26] H. Dannenberg, E. J. M. Speel, J. Zhao et al., "Losses of chromosomes $1 \mathrm{p}$ and $\mathrm{3q}$ are early genetic events in the development of sporadic pheochromocytomas," American Journal of Pathology, vol. 157, no. 2, pp. 353-359, 2000.

[27] E. Edstrom, E. Mahlamaki, B. Nord et al., "Comparative genomic hybridization reveals frequent losses of chromosomes $1 \mathrm{p}$ and $3 \mathrm{q}$ in pheochromocytomas and abdominal paragangliomas, suggesting a common genetic etiology," The American Journal of Pathology, vol. 156, no. 2, pp. 651-659, 2000.

[28] F. H. van Naderveen, E. Korpershoek, R. J. deLeeuw et al., "Array-comparative genomic hybridization in sporadic benign pheochromocytomas," Endocrine-Related Cancer, vol. 16, no. 2, pp. 505-513, 2009.

[29] J. Sandgren, T. Diaz de Stahl, R. Andersson et al., "Recurrent genomic alterations in benign and malignant pheochromocytomas and paragangliomas revealed by whole-genome array comparative genomic hybridization analysis," EndocrineRelated Cancer, vol. 17, no. 3, pp. 561-579, 2010.

[30] D. Astuti, F. Douglas, T. W. J. Lennard et al., "Germline SDHD mutation in familial phaeochromocytoma," The Lancet, vol. 357, no. 9263, pp. 1181-1182, 2001.

[31] C. D. E. Margetts, D. Astuti, D. C. Gentle et al., "Epigenetic analysis of HIC1, CASP8, FLIP, TSP1, DCR1, DCR2, DR4, DR5, KvDMR1, H19 and preferential 11p15.5 maternal-allele loss in von Hippel-Lindau and sporadic phaeochromocytomas," Endocrine-Related Cancer, vol. 12, no. 1, pp. 161-172, 2005.

[32] J. Geli, N. Kiss, M. Karimi et al., "Global and regional CpG methylation in pheochromocytomas and abdominal paragangliomas: association to malignant behavior," Clinical Cancer Research, vol. 14, no. 9, pp. 2551-2559, 2008. 
[33] P. L. M. Dahia, "Pheochromocytoma and paraganglioma pathogenesis: learning from genetic heterogeneity," Nature Reviews Cancer, vol. 14, no. 2, pp. 108-119, 2014.

[34] Y. Aoki, T. Niihori, H. Kawame et al., "Germline mutations in HRAS proto-oncogene cause Costello syndrome," Nature Genetics, vol. 37, no. 10, pp. 1038-1040, 2005.

[35] S. M. Kumar, J. Dai, S. Li et al., "Human skin neural crest progenitor cells are susceptible to BRAF V600E-induced transformation," Oncogene, vol. 33, no. 7, pp. 832-841, 2014.

[36] K. T. Flaherty, I. Puzanov, K. B. Kim et al., "Inhibition of mutated, activated BRAF in metastatic melanoma," The New England Journal of Medicine, vol. 363, no. 9, pp. 809-819, 2010.

[37] D. N. Hayes, A. S. Lucas, T. Tanvetyanon et al., "Phase II efficacy and pharmacogenomic study of selumetinib (AZD6244; ARRY142886) in iodine-131 refractory papillary thyroid carcinoma with or without follicular elements," Clinical Cancer Research, vol. 18, no. 7, pp. 2056-2065, 2012.

[38] D. A. Liebner and M. H. Shah, “Thyroid cancer: pathogenesis and targeted therapy," Therapeutic Advances in Endocrinology and Metabolism, vol. 2, no. 5, pp. 173-195, 2011.

[39] M. Xing, "BRAF mutation in papillary thyroid cancer: pathogenic role, molecular bases, and clinical implications," Endocrine Reviews, vol. 28, no. 7, pp. 742-762, 2007.

[40] S. Seitz, K. Poppe, J. Fischer et al., "Detailed deletion mapping in sporadic breast cancer at chromosomal region $17 \mathrm{p} 13$ distal to theTP53 gene: association with clinicopathological parameters," The Journal of Pathology, vol. 194, pp. 318-326, 2001.

[41] M. Risio, L. Casorzo, L. Chiecchio, G. de Rosa, and F. P. Rossini, "Deletions of 17p are associated with transition from early to advanced colorectal cancer," Cancer Genetics and Cytogenetics, vol. 147, no. 1, pp. 44-49, 2003.

[42] X. Y. Guan, J. S. Sham, L. S. Tai, Y. Fang, H. Li, and Q. Liang, "Evidence for another tumor suppressor gene at 17p13.3 distal to TP53 in hepatocellular carcinoma," Cancer Genetics and Cytogenetics, vol. 140, no. 1, pp. 45-48, 2003.

[43] B.-J. Petri, E.-J. M. Speel, E. Korpershoek et al., "Frequent loss of $17 \mathrm{p}$, but no p53 mutations or protein overexpression in benign and malignant pheochromocytomas," Modern Pathology, vol. 21, no. 4, pp. 407-413, 2008.

[44] T. Yoshimoto, M. Naruse, Z. Zeng et al., "The relatively high frequency of p53 gene mutations in multiple and malignant phaeochromocytomas," Journal of Endocrinology, vol. 159, no. 2, pp. 247-255, 1998.

[45] K. K. Herfarth, M. R. Wick, H. N. Marshall, E. Gartner, S. Lum, and J. F. Moley, "Absence of TP53 alterations in pheochromocytomas and medullary thyroid carcinomas," Genes, Chromosomes and Cancer, vol. 20, no. 1, pp. 24-29, 1997.

[46] B. C. Figueiredo, R. Sandrini, G. P. Zambetti et al., "Penetrance of adrenocortical tumours associated with the germline TP53 R337H mutation," Journal of Medical Genetics, vol. 43, no. 1, pp. 91-96, 2006.

[47] E. Michalkiewicz, R. Sandrini, B. Figueiredo et al., "Clinical and outcome characteristics of children with adrenocortical tumors: a report from the international pediatric adrenocortical tumor registry," Journal of Clinical Oncology, vol. 22, no. 5, pp. 838-845, 2004.

[48] M. I. W. Achatz, M. Olivier, F. L. Calvez et al., "The TP53 mutation, $\mathrm{R} 337 \mathrm{H}$, is associated with $\mathrm{Li}$-Fraumeni and $\mathrm{Li}$ Fraumeni-like syndromes in Brazilian families," Cancer Letters, vol. 245, no. 1-2, pp. 96-102, 2007.
[49] J. G. Assumpção, A. L. Seidinger, M. J. Mastellaro et al., "Association of the germline TP53 R337H mutation with breast cancer in Southern Brazil," BMC Cancer, vol. 8, article 357, 2008.

[50] E. I. Palmero, L. Schuler-Faccini, M. Caleffi et al., "Detection of $\mathrm{R} 337 \mathrm{H}$, a germline TP53 mutation predisposing to multiple cancers, in asymptomatic women participating in a breast cancer screening program in Southern Brazil," Cancer Letters, vol. 261, no. 1, pp. 21-25, 2008.

[51] G. Custodio, G. R. Taques, B. C. Figueiredo et al., "Increased incidence of choroid plexus carcinoma due to the germline TP53 R337H mutation in southern Brazil," PLoS ONE, vol. 6, no. 3, Article ID e18015, 2011.

[52] A. L. Seidinger, M. J. Mastellaro, F. Paschoal Fortes et al., "Association of the highly prevalent TP53 R337H mutation with pediatric choroid plexus carcinoma and osteosarcoma in Southeast Brazil," Cancer, vol. 117, no. 10, pp. 2228-2235, 2011.

[53] H. Yang, D. Ye, K.-L. Guan, and Y. Xiong, "IDH1 and IDH2 mutations in tumorigenesis: mechanistic insights and clinical perspectives," Clinical Cancer Research, vol. 18, no. 20, pp. 55625571, 2012.

[54] J. Gaal, N. Burnichon, E. Korpershoek et al., "Isocitrate dehydrogenase mutations are rare in pheochromocytomas and paragangliomas," The Journal of Clinical Endocrinology \& Metabolism, vol. 95, no. 3, pp. 1274-1278, 2010.

[55] A.-P. Gimenez-Roqueplo, J. Favier, P. Rustin et al., "Mutations in the SDHB gene are associated with extra-adrenal and/or malignant phaeochromocytomas," Cancer Research, vol. 63, no. 17, pp. 5615-5621, 2003.

[56] F. M. Brouwers, G. Eisenhofer, J. J. Tao et al., "High frequency of SDHB germline mutations in patients with malignant catecholamine-producing paragangliomas: implications for genetic testing," Journal of Clinical Endocrinology and Metabolism, vol. 91, no. 11, pp. 4505-4509, 2006.

[57] C. J. Ricketts, J. R. Forman, E. Rattenberry et al., “Tumor risks and genotype-phenotype-proteotype analysis in 358 patients with germline mutations in SDHB and SDHD," Human Mutation, vol. 31, no. 1, pp. 41-51, 2010. 


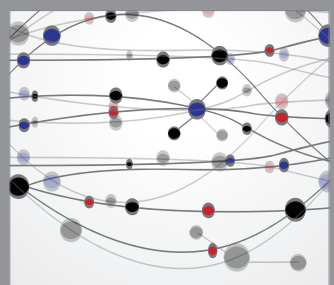

The Scientific World Journal
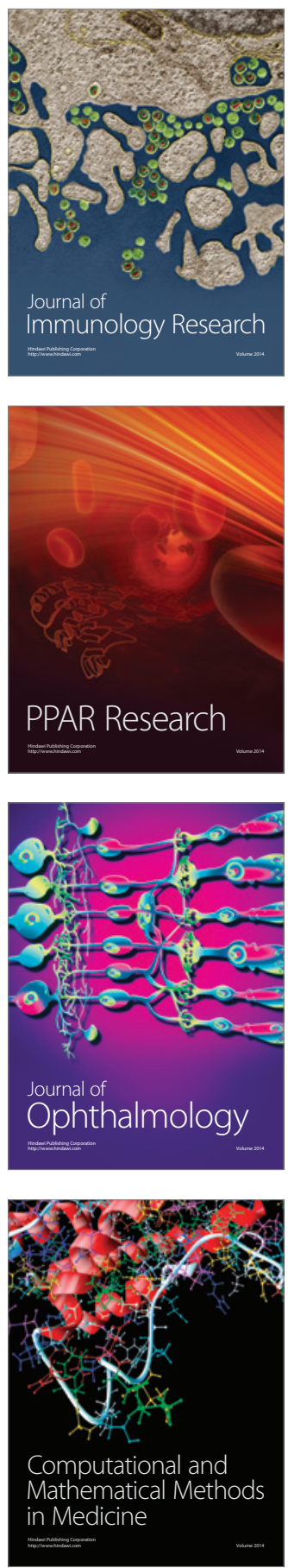

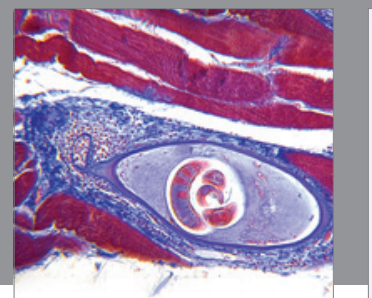

Gastroenterology

Research and Practice
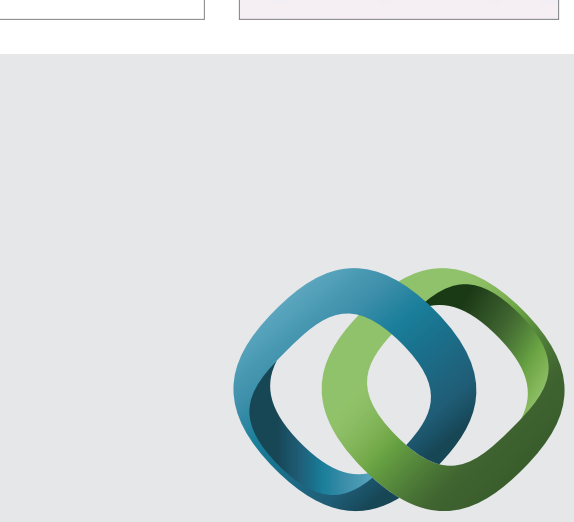

\section{Hindawi}

Submit your manuscripts at

http://www.hindawi.com
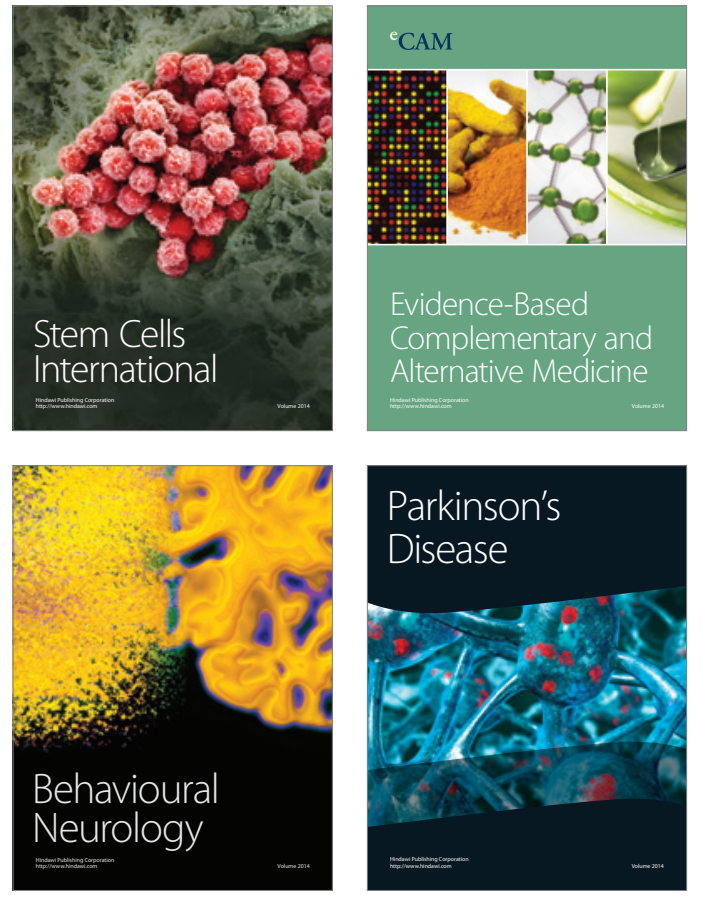
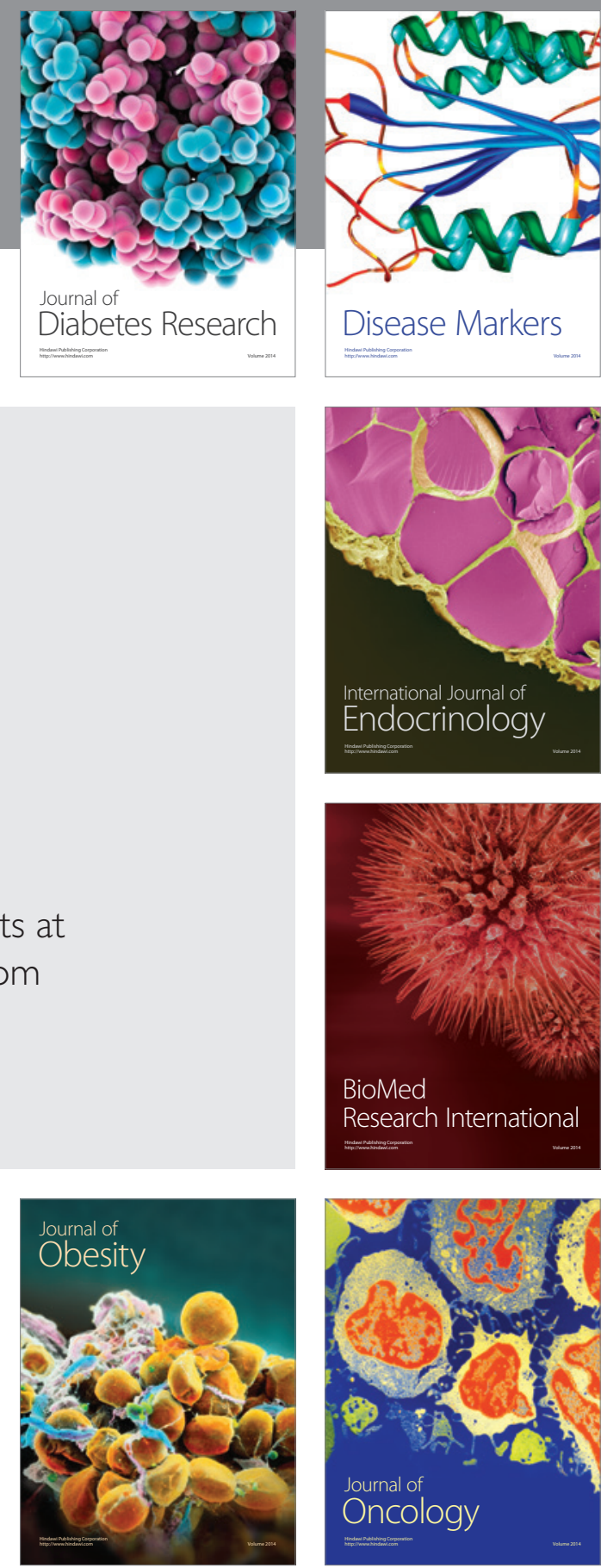

Disease Markers
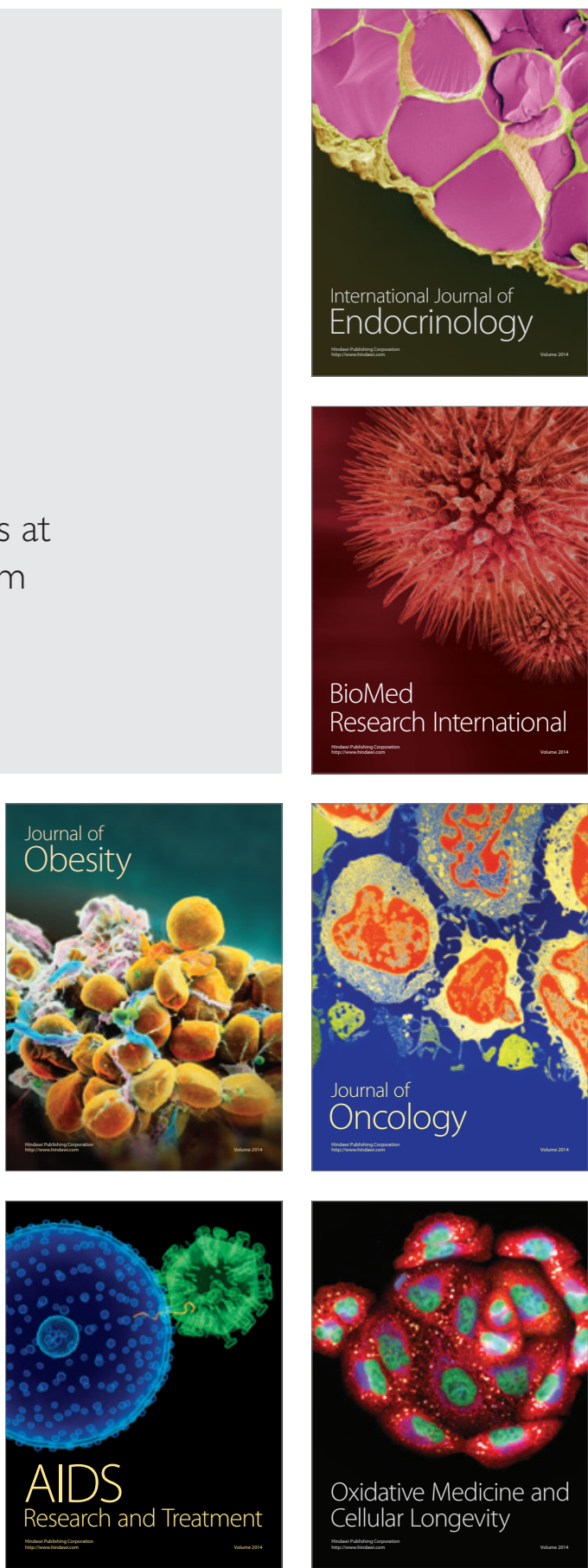